\title{
Physical activity and sarcopenic obesity: definition, assessment, prevalence and mechanism
}

Sarcopenic obesity is the coexistance of sarcopenia and obesity. Modern sarcopenia definition includes low muscle mass, weak muscle strength (handgrip strength) and poor physical function (slow walking), although the clinical definition of each varies worldwide. The cut-points for low muscle mass for men and women using appendicular lean mass divided by height $\left(\mathrm{kg} / \mathrm{m}^{2}\right)$ are $\leq 7.0$ and $\leq 5.4$ in Asians, and $\leq 7.23$ and $\leq 5.67$ in Caucasians, respectively. The cut-points for weak handgrip strength $(\mathrm{kg})$ for men and women are $<26$ and $<18$ in Asians, and $<30$ and $<20$ in Caucasians, respectively. The cut-point for slow walking is $\leq 0.8 \mathrm{~m} / \mathrm{s}$ in men and women. Current data suggest the potential benefits of physical activity and fitness on sarcopenic obesity in older adults.

Lay abstract: Sarcopenic obesity (SO) is the combination of low muscle mass (sarcopenia) and obesity. The average prevalence of SO is about $5-10 \%$. Older adults with SO have higher risks of mobility disability, cardiometabolic diseases and mortality. The medical problems related to SO are much greater than that in sarcopenia or obesity alone. Current studies suggest the potential benefits of physical activity, fitness and resistance exercise on the prevention and treatment of $\mathrm{SO}$ in older adults. This review highlights the importance of the development of effective public health strategies to prevent, delay and treat SO in older people.

First draft submitted: 13 April 2016; Accepted for publication: 11 May 2016; Published online: 14 July 2016

Keywords: exercise $\bullet$ handgrip strength $\bullet$ muscle mass $\bullet$ physical activity $\bullet$ physical fitness

- physical function $\bullet$ sarcopenia $\bullet$ sarcopenic obesity $\bullet$ walking speed

Sarcopenia from the Greek 'sarco' for flesh and 'penia' for loss originally referred to the loss of muscle mass as a natural aging process [1]. In addition, older adults tend to gain fat mass with increasing age with a potential of developing obesity. Based on the national survey data published in 2014, more than one-third (35\%) of American older adults are obese [2]. Additional concern is the rapid growing of the elderly population in most developed countries [3], resulting in a potent epidemiological confluence of risk factors for numerous health-related conditions. Considering that body composition includes both muscle and fat mass, there are four different body composition phenotypes, as described in Figure 1. The combination of high muscle mass and low fat mass is generally considered a healthy combination. Low muscle mass refers to sarcopenia and high fat mass refers to obesity. From a clinical perspective, the most concerning is the combination of low muscle mass plus high fat mass, termed sarcopenic obesity (SO) due to the coexistence of sarcopenia and obesity. SO is more common in older adults than young adults due to the natural changes in body composition associated aging.
Duck-chul Lee ${ }^{*, 1}$, Robin P Shook', Clemens Drenowatz ${ }^{2}$ \& Steven N Blair ${ }^{2,3}$

'Department of Kinesiology, College of Human Sciences, lowa State University, Ames, IA 50011, USA

${ }^{2}$ Department of Exercise Science, University of South Carolina, Columbia, SC 29208, USA

${ }^{3}$ Department of Epidemiology \& Biostatistics, University of South Carolina, Columbia, SC 29208, USA *Author for correspondence: Tel.: +1 5152948042 Fax: +1 5152948740 dclee@iastate.edu 


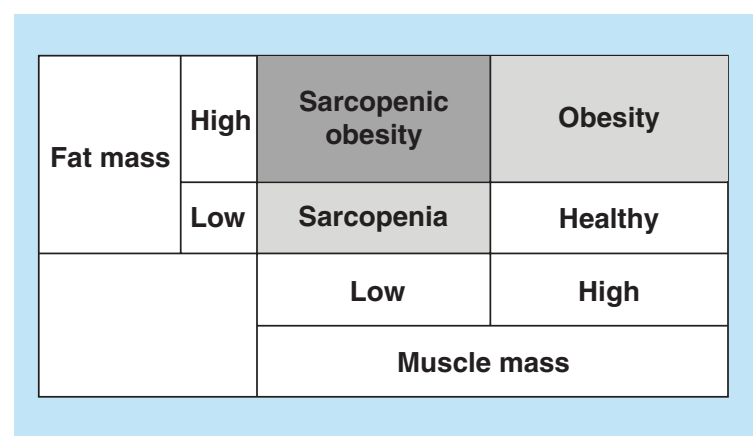

Figure 1. Sarcopenic obesity by body composition phenotype.

Compelling evidence has shown that older adults with SO have higher risks of mobility disability [4-6], metabolic diseases [7,8], hypertension [9], cardiovascular diseases [10,11] and mortality [12-14], which is also related to significantly higher healthcare costs [15]. These clinical problems related to $\mathrm{SO}$ are much greater than that in sarcopenia or obesity alone $[4,9,12,13]$, which suggests that sarcopenia and obesity have independent and additive adverse effects on health in the elderly. However, despite this significant and rising public health concern, there is very little evidence on SO. Therefore, investigating and developing effective $\mathrm{SO}$ prevention and treatment programs should be a priority based on the dramatically increasing health impact of $\mathrm{SO}$ in aging populations.

Although a certain degree of muscle loss and fat gain due to aging is inevitable, the good news is that $\mathrm{SO}$ is a modifiable condition, thus could be prevented and treated following effective therapy. Among related factors to $\mathrm{SO}$, physical activity has been recognized as a key lifestyle factor to prevent and delay muscle loss and obesity with aging [16-18]. However, a few data are currently available on the effects of physical activity on $\mathrm{SO}$ in older adults, although there is accumulating evidence suggesting health benefits of physical activity on either sarcopenia or obesity. One of the major reasons of the limited data on SO is due to the differences in the definition and assessment of sarcopenia and SO among various working groups on sarcopenia. Also, data on the possible mechanisms of $\mathrm{SO}$ in various health conditions and the role of physical activity on the development of SO are still lacking. Therefore, the current review focuses specifically on SO including its clinical definition, assessment, diagnostic criteria, prevalence and potential mechanisms. We also explored and summarized the associations of physical activity, physical fitness and exercise training with $\mathrm{SO}$ in older adults.

\section{Method}

In this review on physical activity and SO, we used PubMed to identify relevant peer-reviewed journal articles (the last search was conducted on 18 December 2015). The search keywords included 'physical activity' or 'exercise' or 'fitness' and 'sarcopenic obesity' using the advanced search method. We included all original human studies from cross-sectional surveys, observational cohorts and clinical trials that are not limited by publication year, population age, gender or country. However, conference abstracts, review articles, editorials or commentaries were not included. A total of 96 articles were initially identified. We read each abstract and further reviewed references from selected and eligible articles for additional research. To be included in the quantitative analyses, we required data on risk ratios (odds ratios or relative risk) in observational studies or changes in $\mathrm{SO}$ variables in intervention studies, comparing active or exercise groups against inactive or control groups. We found a total of six original research articles that investigated the associations of physical activity, fitness or exercise with SO; five cross-sectional studies and one randomized controlled trial. In addition to study design, the characteristics of the selected studies were further assessed by study population, measurement of physical activity, fitness or exercise program, definitions of sarcopenia and obesity and the prevalence of SO. The data extracted from the selected studies are presented in Table 1 for comparisons among studies.

\section{Assessment \& definition of sarcopenic obesity}

As mentioned previously, there currently is no universally adopted definition of SO, primarily due to the variations in the definition of sarcopenia (Table 2). Modern definitions of sarcopenia as a progressive medical condition includes not only loss of muscle mass, but also weakness of muscle strength (handgrip strength) and/or poor physical function (performance), which are well-established health predictors. Handgrip strength is a strong predictor of all-cause and cardiovascular mortality in people of diverse economic and sociocultural backgrounds based on a large longitudinal population study conducted in 17 countries [25]. The common physical function test, gait speed, is a significant predictor of disability [26] and survival [27]. In addition, there is supporting evidence indicating stronger associations of muscle strength with mobility limitations in older adults [28], but weak or no associations of muscle mass alone with mortality [29]. These data support the concept that consideration should be made of all three sarcopenia criteria: muscle mass, muscle strength and physical function.

\section{Assessment of sarcopenia Muscle mass}

In recent studies, body composition to measure muscle mass was most commonly acquired from whole body 


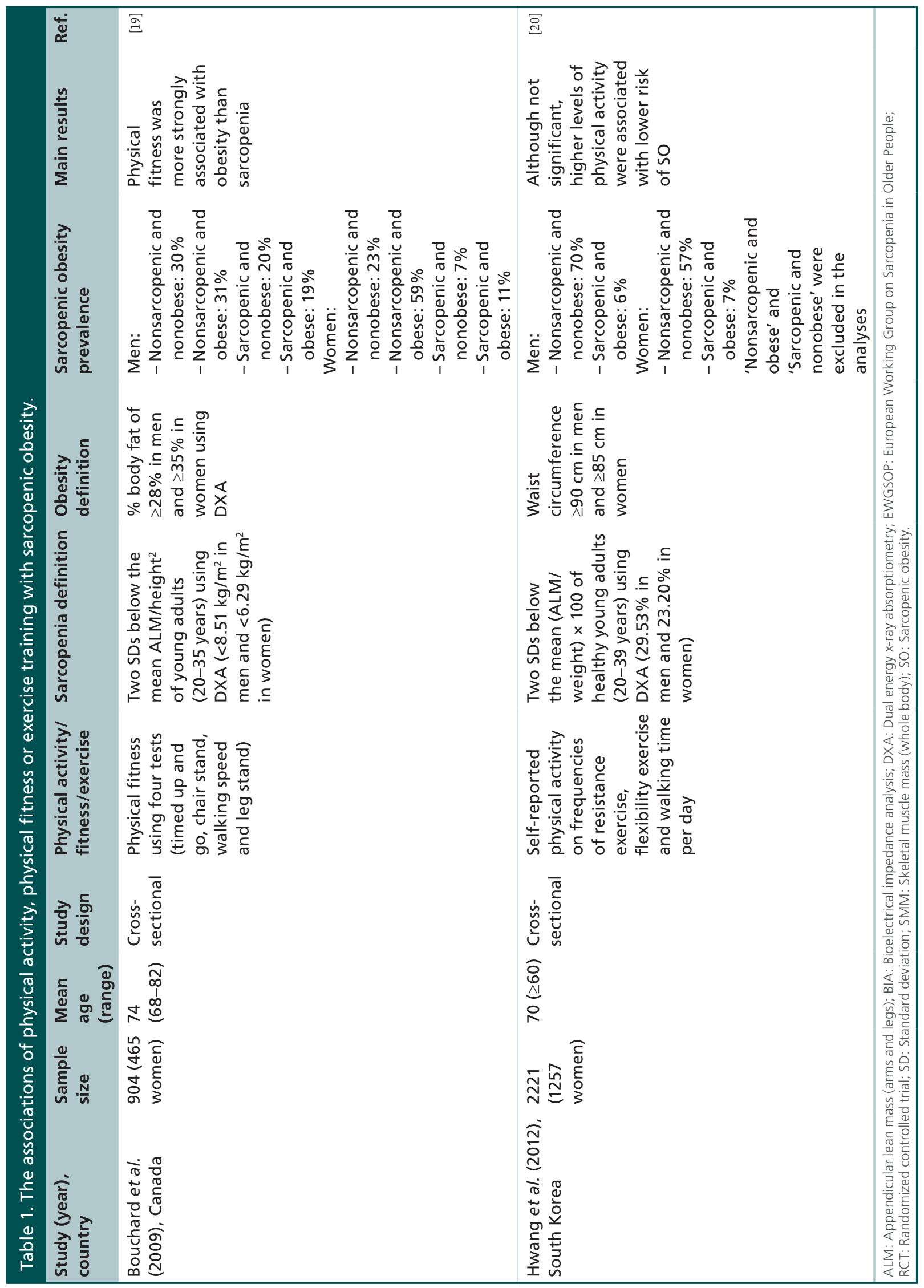




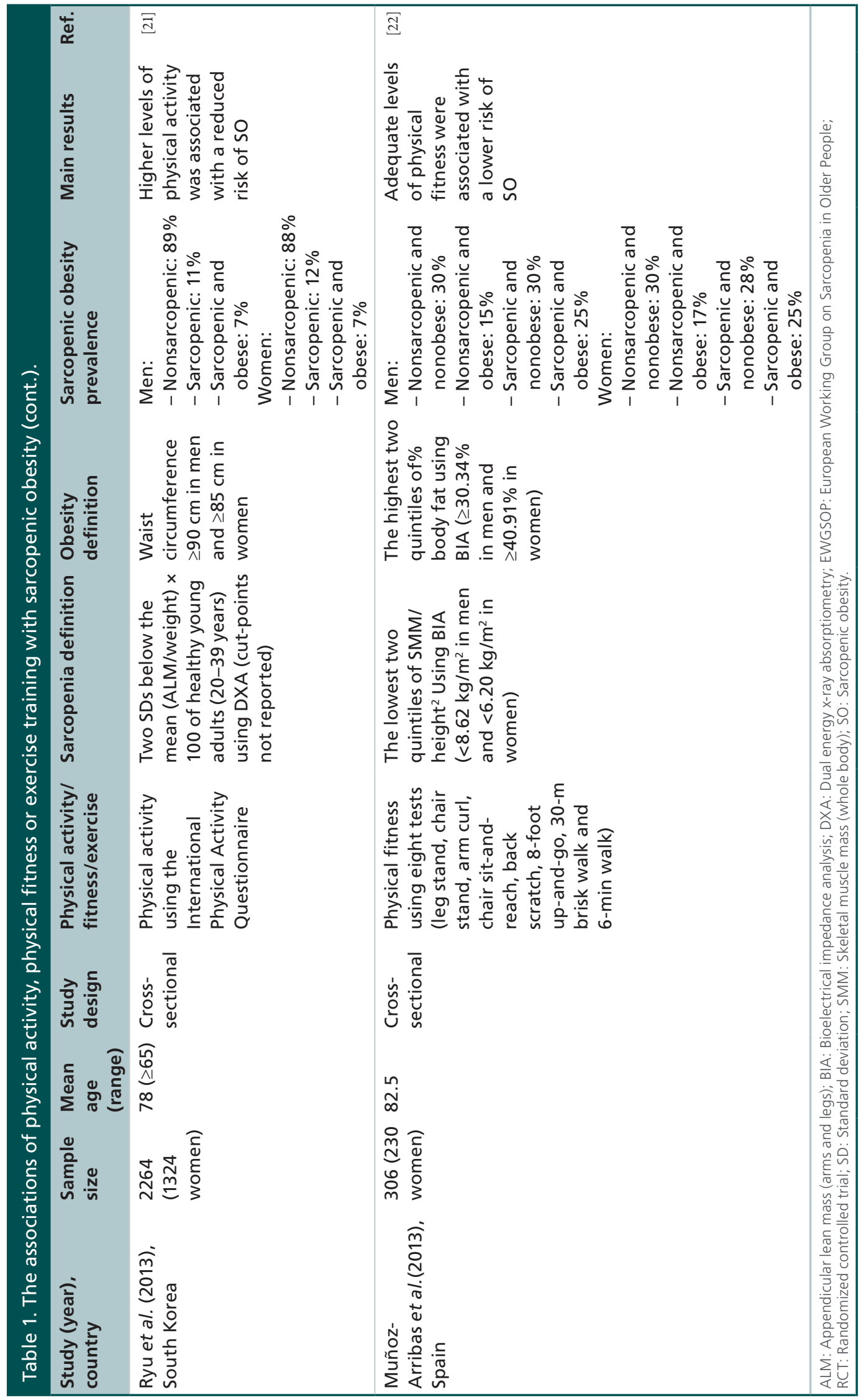




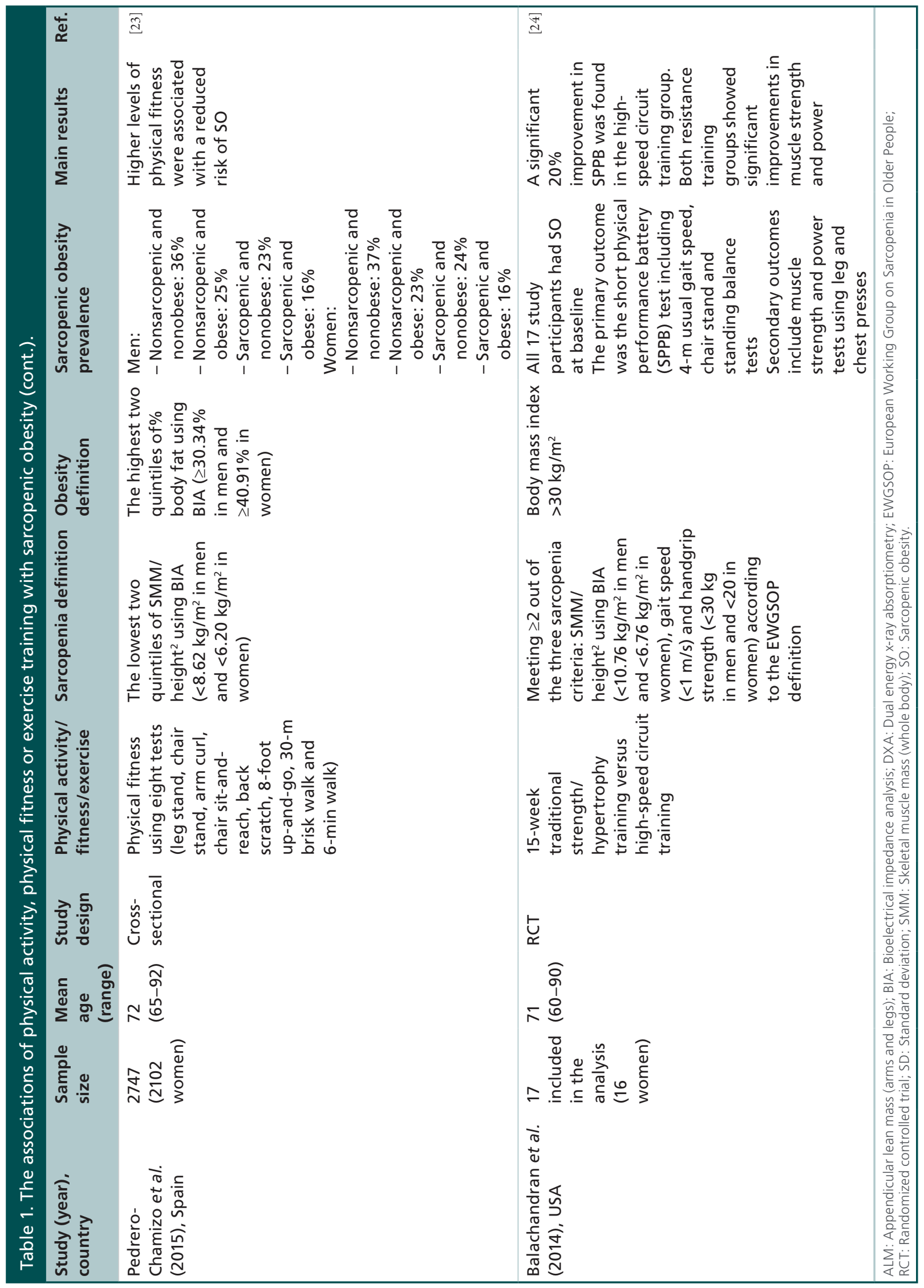


Table 2. Clinical definition of sarcopenia.

\begin{tabular}{|c|c|c|c|c|c|c|}
\hline \multirow[t]{2}{*}{ Group } & \multicolumn{5}{|c|}{ Diagnostic criteria and cut-points for sarcopenia } & \multirow[t]{2}{*}{ Ref. } \\
\hline & Physical function & & Muscle strength & & Muscle mass & \\
\hline \multirow{8}{*}{$\begin{array}{l}\text { European Working Group on Sarcopenia } \\
\text { in Older People (EWGSOP, 2010) }\end{array}$} & \multirow[t]{8}{*}{ Gait speed $\leq 0.8 \mathrm{~m} / \mathrm{s}$} & \multirow[t]{8}{*}{ Or } & Handgrip strength & \multirow[t]{8}{*}{ And } & DXA ALM/height ${ }^{2}$ & \multirow[t]{8}{*}{ [30] } \\
\hline & & & Men: $<30 \mathrm{~kg}$ & & Men: $\leq 7.23 \mathrm{~kg} / \mathrm{m}^{2}$ & \\
\hline & & & Women: $<20$ kg & & Women: $\leq 5.67 \mathrm{~kg} / \mathrm{m}^{2}$ & \\
\hline & & & & & BIA SMM/height ${ }^{2}$ & \\
\hline & & & & & Men: $<8.87 \mathrm{~kg} / \mathrm{m}^{2 \dagger}$ & \\
\hline & & & & & Women: $<6.42 \mathrm{~kg} / \mathrm{m}^{2 \dagger}$ & \\
\hline & & & & & Men: $<10.76 \mathrm{~kg} / \mathrm{m}^{2 \ddagger}$ & \\
\hline & & & & & Women: $<6.76 \mathrm{~kg} / \mathrm{m}^{2 \ddagger}$ & \\
\hline \multirow{3}{*}{$\begin{array}{l}\text { International Working Group on } \\
\text { Sarcopenia (IWGS, 2011) }\end{array}$} & \multirow{3}{*}{\multicolumn{2}{|c|}{ Gait speed $<1.0 \mathrm{~m} / \mathrm{s}$}} & & \multirow[t]{3}{*}{ And } & DXA ALM/height ${ }^{2}$ & \multirow[t]{3}{*}{ [31] } \\
\hline & & & & & Men: $\leq 7.23 \mathrm{~kg} / \mathrm{m}^{2}$ & \\
\hline & & & & & Women: $\leq 5.67 \mathrm{~kg} / \mathrm{m}^{2}$ & \\
\hline \multirow{6}{*}{$\begin{array}{l}\text { Asian Working Group for Sarcopenia } \\
\text { (AWGS, 2014) }\end{array}$} & \multirow[t]{6}{*}{ Gait speed $\leq 0.8 \mathrm{~m} / \mathrm{s}$} & \multirow[t]{6}{*}{ Or } & Handgrip strength & \multirow[t]{6}{*}{ And } & DXA ALM/height ${ }^{2}$ & \multirow[t]{6}{*}{ [32] } \\
\hline & & & Men: $<26$ kg & & Men: $\leq 7.0 \mathrm{~kg} / \mathrm{m}^{2}$ & \\
\hline & & & \multirow[t]{4}{*}{ Women: $<18 \mathrm{~kg}$} & & Women: $\leq 5.4 \mathrm{~kg} / \mathrm{m}^{2}$ & \\
\hline & & & & & BIA ALM/height ${ }^{2 \S}$ & \\
\hline & & & & & Men: $\leq 7.0 \mathrm{~kg} / \mathrm{m}^{2}$ & \\
\hline & & & & & Women: $\leq 5.7 \mathrm{~kg} / \mathrm{m}^{2}$ & \\
\hline \multirow{3}{*}{$\begin{array}{l}\text { Foundation for the NIH Sarcopenia } \\
\text { Project (FNIHSP, 2014) }\end{array}$} & \multirow[t]{3}{*}{ Gait speed $\leq 0.8 \mathrm{~m} / \mathrm{s}$} & \multirow[t]{3}{*}{ And } & Handgrip strength & \multirow[t]{3}{*}{ And } & DXA ALM/BMI & \multirow[t]{3}{*}[33]{} \\
\hline & & & Men: $<26 \mathrm{~kg}$ & & Men: $<0.789$ & \\
\hline & & & Women: <16 kg & & Women: $<0.512$ & \\
\hline
\end{tabular}

dual-energy x-ray absorptiometry (DXA). For skeletal muscle mass (SMM), appendicular lean mass (ALM) is generally used, which is calculated as the sum of lean mass in arms and legs without including fat and bone mass using DXA. ALM is also referred to as appendicular SMM or appendicular fat free mass. To define sarcopenia, relative muscle mass is typically utilized because absolute muscle mass is highly correlated with height or weight. Similar to body mass index calculation (total body weight divided by height squared, $\mathrm{kg} / \mathrm{m}^{2}$ ), most sarcopenia definitions utilized the ALM index, ALM in $\mathrm{kg}$ divided by height squared in meter $(\mathrm{kg} /$ $\mathrm{m}^{2}$ ) [30-32] or divided by body mass index (BMI) [33], as a ratio of muscle mass to height or BMI. The cutpoint of the height adjusted ALM index for low muscle mass was mostly established as two SD below the mean value of sex-specific reference values in healthy young adults (18-40 years), which was originally proposed by Baumgartner et al. [36]. One of the limitations of using ALM from DXA is that ALM does not include trunk muscles such as chest and back muscles, one of the largest muscle groups in humans. It is because the trunk has body organs (heart, lung and intestines) and DXA cannot accurately distinguish these from muscle mass. However, because people use their arms and legs in most daily activities as well as during resistance exercise to develop chest and back muscles, it is likely that trunk muscle mass is highly correlated to ALM. Therefore, using ALM from DXA is reasonable, although using total body lean mass including trunk muscles is ideal in future studies with more advanced technologies for total body composition assessment [37]. Also, based on its accuracy and availability, DXA is useful and practical for research and clinical use. Further, DXA has relatively low radiation compared with other imaging technologies such as computed tomography (CT) or MRI, which are more precise, yet expensive and have more radiation exposure. However, all these imaging technologies and equipment (DXA, CT and MRI) are not portable, which is important in large epidemiological studies in populations. Therefore, more research is needed to define sarcopenia 
and $\mathrm{SO}$ using other traditional or modern technologies that are safe, inexpensive and widely available including anthropometry (e.g., midupper arm or calf circumference) [38,39], bioelectrical impedance analysis (BIA) [40] and ultrasound [37].

\section{Muscle strength}

Handgrip (grip) strength has been widely used for muscle strength measurement, because it is inexpensive, easy to use and well correlated with most relevant health outcomes such as mortality [25]. Handgrip strength is measured by a handheld dynamometer, mostly using Jamar dynamometer and the maximum value from either hand or combined from both hands are analyzed [30]. However, because leg strength is more related to physical functions such as gait, chair standing and stair climbing, leg strength tests also have been utilized, especially in research studies. Common leg strength tests are knee flexion and extension at various velocities using isokinetic equipment (e.g., Biodex). This isokinetic test measures muscle power (work per unit time), which is the capacity of muscles to rapidly exert force as a measure of the explosiveness of the muscle. Measuring power may also be important in sarcopenia research in older adults because it is suggested that power could be a better predictor of functional capacities, as power is lost faster than strength during aging [41,42]. Another strength test is the one repetition maximum (1-RM) test, commonly using bench and/or leg presses. This test is traditionally used by athletic or fitness trainers to evaluate training programs. However, there is also strong evidence indicating health benefits of increased total body muscle strength measured by $1-R M$ test in general populations [43-45]. Nevertheless, because isokinetic and 1-RM tests need special equipment and proper training, they are less practical in clinical use. Therefore, more feasible as well as reliable lower body and total body strength tests, which are highly correlated with physical function, should be continuously developed and validated.

\section{Physical function}

There are a wide range of physical function tests, including usual gait speed, $6 \mathrm{~min}$ or 400 -m walk tests, timed get-up-and-go test, chair stand test and the short physical performance battery, which is a composite measure of balance, gait and leg strength. However, usual gait speed is the most popular physical function and performance test in clinical practice and sarcopenia research because it is simple, fast and easy to measure as a predictor of mobility limitations and mortality in general populations, as well as patients after cardiac surgery [46-48]. Habitual gait speed was mostly measured at the usual pace on a 4- or 6-m course, and the average or the best value was used. Inability to rise from a chair was also considered as an alternate definition of mobility disability $[30,31]$.

\section{Definitions \& prevalence of sarcopenia}

Because sarcopenia no longer refers purely to loss of muscle mass, there are differences in how sarcopenia is defined. Table 2 shows various clinical definitions of sarcopenia by major professional organizations and groups on sarcopenia.

The European Working Group on Sarcopenia in Older People (EWGSOP) recommends using the presence of low muscle mass plus either low muscle strength or poor physical function for the diagnosis of sarcopenia [30]. They noted that defining sarcopenia using only muscle mass is too narrow and may be of limited clinical value based on the fact that muscle strength does not depend solely on muscle mass and the relationship between strength and mass is not linear [49]. They further defined conceptual stages as 'presarcopenia' (low muscle mass), 'sarcopenia' (low muscle mass plus either low muscle strength or poor physical function) and 'severe sarcopenia' (low muscle mass, low muscle strength and poor physical function) based on the single or combination of three sarcopenia criteria to help select appropriate treatment and recovery goals. To define sarcopenia, the EWGSOP recommends a cut-point at two SDs below the mean value of healthy young reference adults. To identify sarcopenia cases, they suggest initially measuring gait speed, as it is considered the easiest and most reliable assessment, using the cut-point value of $\leq 0.8 \mathrm{~m} / \mathrm{s}$ (slow walkers). Then, assessments of either handgrip strength or muscle mass should be followed based on walking speed. For slow walkers, the muscle mass assessment is then completed to define sarcopenia. For fast walkers, the handgrip strength test is additionally suggested to define low muscle strength, then final muscle mass was assessed. Therefore, in both cases, low muscle mass in addition to poor physical function and/or poor handgrip strength is necessary to be diagnosed with sarcopenia. In terms of numeric definitions, the EWGSOP recommends several cut-points for muscle mass based on different studies and measurement techniques. Among those different cut-points for muscle mass, we selected ALM index measured using DXA of $7.23 \mathrm{~kg} / \mathrm{m}^{2}$ for men and $5.67 \mathrm{~kg} / \mathrm{m}^{2}$ for women, which is the same cut-point recommended by the International Working Group on Sarcopenia (IWGS) 1 year later [31]. These cut-points were also used in another study to compare different sarcopenia definitions [50]. Also, the EWGSOP provides two other cut-points for low total body SMM measured by BIA, including cut-points of $<8.87 \mathrm{~kg} / \mathrm{m}^{2}$ for men and $<6.42 \mathrm{~kg} / \mathrm{m}^{2}$ for women, which were vali- 
dated by comparing with MRI [34]. The other provided BIA-based cut-points were $<10.76 \mathrm{~kg} / \mathrm{m}^{2}$ for men and $<6.76 \mathrm{~kg} / \mathrm{m}^{2}$ for women, which were developed based on a large US national sample of 4449 older adults [35].

In the sarcopenia definition by the IWGS, the diagnosis of sarcopenia was given to individuals with poor physical function plus low muscle mass, without considering handgrip strength [31]. Poor physical function is defined as individuals who are bedridden, nonambulatory, cannot independently rise from a chair or who have a measured gait speed $<1.0 \mathrm{~m} / \mathrm{s}$ (most easily identifiable measure). These individuals with poor physical function should be further examined for muscle mass. Low muscle mass is defined by ALM index of $\leq 7.23 \mathrm{~kg} / \mathrm{m}^{2}$ in men and $\leq 5.67 \mathrm{~kg} / \mathrm{m}^{2}$ in women using DXA. These cut-points of ALM index for sarcopenia were developed based on the sex-specific lowest $20 \%$ of the distribution of the ALM index from the Health ABC Study (3075 well-functioning men and women aged 70-79 recruited in 1997-1998 from a random sample of Medicare enrollees in Pennsylvania and Tennessee in USA) [51].

The Asian Working Group for Sarcopenia (AWGS) supports using both DXA and BIA to define low muscle mass for sarcopenia diagnosis [32]. They indicated that although DXA may be the most widely used method for muscle mass assessment in sarcopenia studies, BIA is suitable based on its portability, reasonable cost, fast processing, noninvasiveness, radiation-free functions and convenience, especially in communitybased screening programs. They provide evidence suggesting a reliable estimation of ALM from BIA, using DXA as a reference method in community-dwelling Japanese older women [52]. In another study in elderly Taiwanese men, results of fat-free mass using BIA were also associated with the results using DXA [53]. The AWGS recommends using two SDs below the mean muscle mass of a young reference group or the lower quintile as the cut-point value. They also recommend using height-adjusted ALM index, and cut-points for low muscle mass as $7.0 \mathrm{~kg} / \mathrm{m}^{2}$ in men and $5.4 \mathrm{~kg} / \mathrm{m}^{2}$ in women by using DXA, and $7.0 \mathrm{~kg} / \mathrm{m}^{2}$ in men and $5.7 \mathrm{~kg} / \mathrm{m}^{2}$ in women by using BIA. However, the cutpoints in Asians are lower than those of Caucasian populations using the same DXA, recommended by the EWGSOP and IWGS groups on sarcopenia. This is because of relatively low prevalence of sarcopenia in Asian studies, which is partly due to lower body weight in Asians, compared with Caucasians. There are also differences in socioeconomic factors, lifestyle and culture between Asians and Caucasians. Regarding muscle strength, most Asian sarcopenia research studies used handgrip strength, the AWGS also recommends it as a feasible and convenient measure, similar to other sarcopenia definitions. The cut-point values of low handgrip strength are suggested to be defined as $26 \mathrm{~kg}$ in men and $18 \mathrm{~kg}$ in women. Regarding physical function, the AWGS recommends using a 6-m usual walking speed. After extensive review of Asian data, slow walking speed was defined as $\leq 0.8 \mathrm{~m} / \mathrm{s}$, which is consistent with the EWGSOP and the US definitions below. However, they reported that there is a potential gender difference in the cut-point value of usual walking speed with a wide range from 0.6 to $1.2 \mathrm{~m} / \mathrm{s}$.

In the US Foundation for the National Institutes of Health Sarcopenia Project (FNIHSP), a total of 26,625 participants (11,427 men and 15,198 women) were included in the pooled data analyses from nine studies after excluding participants $<65$ years old [33]. This is one of the largest sarcopenia projects incorporating diverse populations from different races, ethnicities, geographic regions and a range of health and functional status. The average age was 75.2 years for men and 78.6 years for women. The average BMIs were $27.1 \mathrm{~kg} / \mathrm{m}^{2}$ in men and $26.9 \mathrm{~kg} / \mathrm{m}^{2}$ in women. They recommended sequential screening and case identification processes: screening for physical function first using gait speed, followed by strength assessment using handgrip strength and then assessment of lean mass using DXA. The FNIHSP selected a usual gait speed $\leq 0.8 \mathrm{~m} / \mathrm{s}$ for poor physical function, which is the same cut-point suggested by the EWGSOP and the AWGS groups. They reported that $10 \%$ of men and $31 \%$ of women had gait speed $\leq 0.8 \mathrm{~m} / \mathrm{s}$. The final recommended cut-points for muscle weakness were handgrip strength $<26 \mathrm{~kg}$ for men and $<16 \mathrm{~kg}$ for women. Regarding muscle mass assessment, experts in the study preferred a measure that accounts for body mass index instead of height that was suggested earlier in other sarcopenia groups. The final recommended cutpoints of ALM/BMI index for low muscle mass using DXA were $<0.789$ for men and $<0.512$ for women.

Based on a review of the recommendations by four major working groups on sarcopenia above, and a careful examination of the existing literature, we propose the following recommendations (Table 3).

First, slow walking (poor physical function) was most commonly defined as gait speed of $\leq 0.8 \mathrm{~m} / \mathrm{s}$ in both men and women regardless of race and ethnicity. This cut-point is supported by the European, Asian and the US groups. For muscle strength and mass, it is more appropriate to use separate cut-points for Asians and Caucasians based on higher adiposity in Asians given the same body weight [54], lower handgrip strength in Asians [25], lower prevalence of sarcopenia in Asians [32] and different lifestyle and cultural factors between Asians and Caucasians of European origin. This approach is also in line with different cut-points 
Table 3. Recommendation for the diagnostic criteria and cut-points for sarcopenia.

\begin{tabular}{|c|c|c|c|c|c|}
\hline Population & $\begin{array}{l}\text { Physical function } \\
\text { (gait speed) }\end{array}$ & & $\begin{array}{l}\text { Muscle strength } \\
\text { (handgrip strength) }\end{array}$ & & 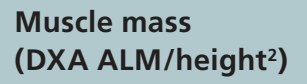 \\
\hline \multirow[t]{2}{*}{ Asian } & Men: $\leq 0.8 \mathrm{~m} / \mathrm{s}$ & Or & Men: <26 kg & And & Men: $\leq 7.00 \mathrm{~kg} / \mathrm{m}^{2}$ \\
\hline & Women: $\leq 0.8 \mathrm{~m} / \mathrm{s}$ & & Women: $<18 \mathrm{~kg}$ & & Women: $\leq 5.40 \mathrm{~kg} / \mathrm{m}^{2}$ \\
\hline \multirow[t]{2}{*}{ Caucasian } & Men: $\leq 0.8 \mathrm{~m} / \mathrm{s}$ & Or & Men: $<30 \mathrm{~kg}$ & And & Men: $\leq 7.23 \mathrm{~kg} / \mathrm{m}^{2}$ \\
\hline & Women: $\leq 0.8 \mathrm{~m} / \mathrm{s}$ & & Women: <20 kg & & Women: $\leq 5.67 \mathrm{~kg} / \mathrm{m}^{2}$ \\
\hline
\end{tabular}

for obesity between Asians (BMI $\geq 25 \mathrm{mg} / \mathrm{m}^{2}$ ) and Caucasians (BMI $\geq 30 \mathrm{mg} / \mathrm{m}^{2}$ ), suggested by the World Health Organization [55]. The recommended cutpoints for low handgrip strength (muscle weakness) are $<26 \mathrm{~kg}$ in men and $<18 \mathrm{~kg}$ in women in Asians, as suggested by the Asian group and $<30 \mathrm{~kg}$ in men and $<20 \mathrm{~kg}$ in women in Caucasians, as suggested by the European group. However, the US group suggested lower cut-points ( $<26 \mathrm{~kg}$ in men and $<16 \mathrm{~kg}$ in women) for low handgrip strength than that by the European group. Based on the lower levels of handgrip strength in Asians [25], using the higher cut-points for weak handgrip strength in Caucasians suggested by the European group would be reasonable. However, more studies are needed to identify universal cut-points for low handgrip strength in Caucasians, which should be predictable of health outcomes such as functional disability and mortality. Regarding low muscle mass to define sarcopenia, we recommend cut-points of ALM/height ${ }^{2}$ $\leq 7.0 \mathrm{~kg} / \mathrm{m}^{2}$ in men and $\leq 5.4 \mathrm{~kg} / \mathrm{m}^{2}$ in women using DXA in Asians, as suggested by the Asian group. For Caucasians, ALM/height ${ }^{2}$ of $\leq 7.23 \mathrm{~kg} / \mathrm{m}^{2}$ in men and $\leq 5.67 \mathrm{~kg} / \mathrm{m}^{2}$ in women using DXA were suggested by European and international groups. However, BMI adjusted ALM cut-points of $<0.789$ in men and $<0.512$ in women could also be used in Caucasians, as suggested by the US group. Also, in large population studies when DXA is not available, BIA-based cut-points for low muscle mass shown in Table 2 could be a practical option. All four major working groups on sarcopenia recommend low muscle mass as a core diagnostic criteria for sarcopenia. However, there is an inconsistency regarding other diagnostic criteria for sarcopenia. The European and Asian groups recommend that either slow walking speed or low handgrip strength should additionally be included for sarcopenia diagnosis, whereas the US group suggests to include both slow walking speed and low handgrip strength in addition to low muscle mass. On the other hand, the IWGS includes only slow walking speed, but not muscle strength, in their diagnostic criteria for sarcopenia in addition to low muscle mass. Therefore, more studies are needed whether using all three criteria or which two criteria increases diagnostic power. At this point, we recommend to include at least either slow walking speed or low handgrip strength in addition to low muscle mass based on the current recommendations by the European and Asian groups.

A recent study compared sarcopenia prevalence using different definitions based on data from nine studies in an older adults population aged $\geq 65$ years old (a total of 7113 men and 2950 women were included in the analyses) [50]. They found that the prevalence of sarcopenia was higher in women than men. They also found lower prevalence of sarcopenia with the US definition (1.3\% in men and $2.3 \%$ in women), compared with the European definition $(5.3 \%$ in men and $13.3 \%$ in women) and Asian definition (5.1\% in men and $11.8 \%$ in women). Therefore, BMI adjusted ALM compared with height adjusted ALM in defining sarcopenia may result in a more conservative definition. These different definitions, even in the same Caucasian populations, make it difficult to compare results between studies. Therefore, it is important to develop a universal criterion and definition for the diagnosis of sarcopenia.

\section{Definition \& prevalence of sarcopenic obesity}

Theoretically, SO has been narrowly defined by low muscle mass and high fat mass, as described earlier in Figure 1. However, recent SO studies have used an expanded diagnostic criteria for identifying both sarcopenia and obesity, which also incorporates single or combination of different assessments of sarcopenia and the quantification of both systemic and central adiposity (Figure 2).

In addition, the cut-point values of each sarcopenia and obesity criterion are also different between studies, depending on population, gender, age, race and ethnicity. Most studies used ALM index divided by height ${ }^{2}<2.0$ SDs $[4,5,19,56]$ or ALM index divided by weight $\leq 2.0$ SDs $[8,20,21,57]$ using DXA to define sarcopenia. However, other studies used the lowest two quintiles of SMM divided by height ${ }^{2}$ using BIA [22,23], handgrip strength lowest tertile [58,59] or walking speed $\leq 0.8 \mathrm{~m} / \mathrm{s}$ [60]. To define obesity, some studies used BMI $\geq 30 \mathrm{~kg} / \mathrm{m}^{2}$ in men and women [51,59] 


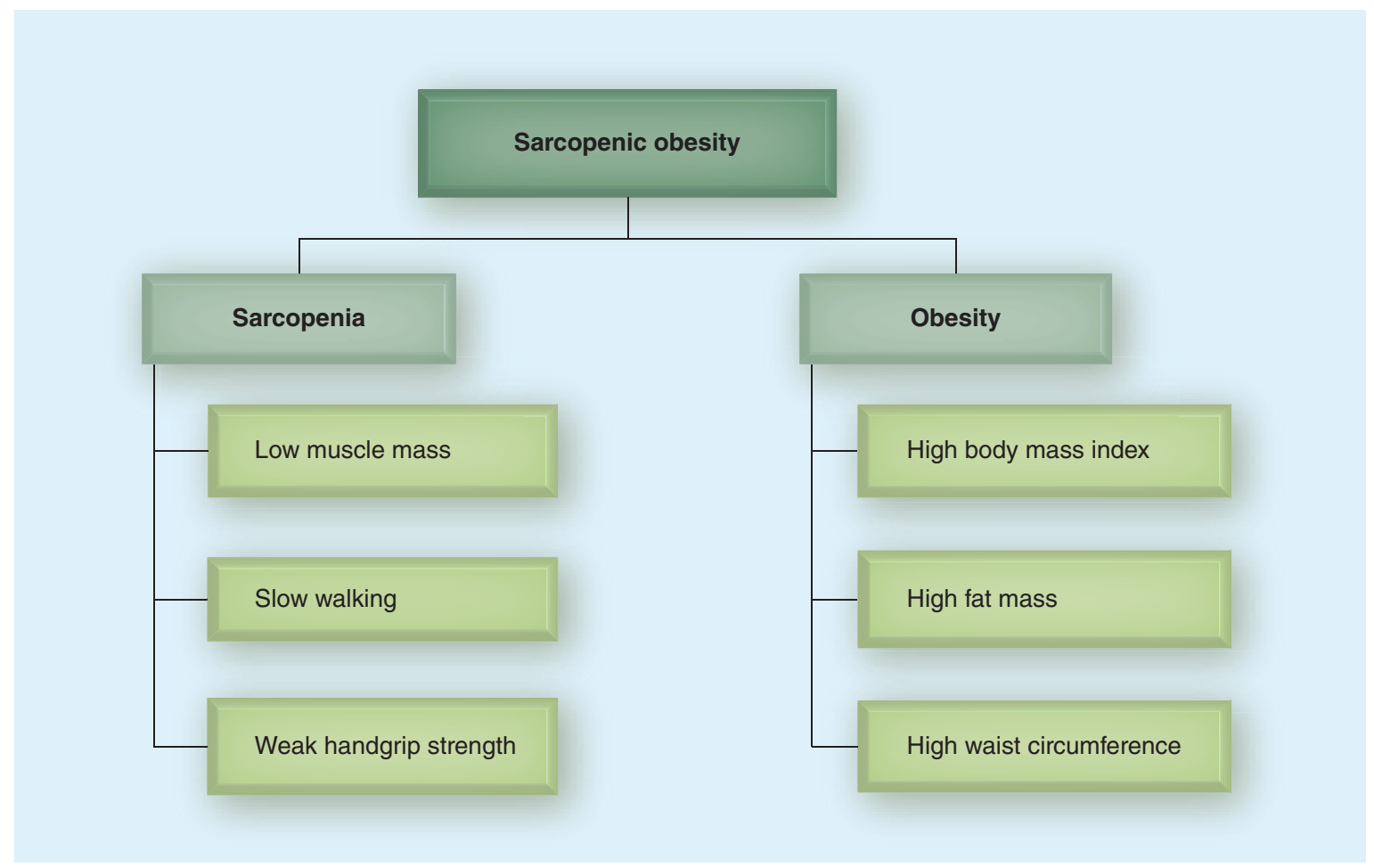

Figure 2. Diagnostic criteria for sarcopenic obesity.

or BMI $>27.5 \mathrm{~kg} / \mathrm{m}^{2}$ in men [60], and others used different \% body fat values of $>27 \%$ in men and $>38 \%$ in women [4], $>28 \%$ in men and $>35 \%$ in women [19], $>30 \%$ in men and $>40 \%$ in women $[5,61]$ or the highest two quintiles of $\%$ body fat $[22,23]$. Instead of BMI or $\%$ body fat, some studies also used visceral fat area $>100 \mathrm{~cm}^{2}$ by CT scan [8], or waist circumference upper tertile [58], $\geq 90 \mathrm{~cm}$ in men and $\geq 85 \mathrm{~cm}$ in women $[20,21,57]$ or $>102 \mathrm{~cm}$ in men and $>88 \mathrm{~cm}$ in women [56]. Due to these various cut-point values of sarcopenia and obesity, comparing findings between studies are challenging. Also, the prevalence of $\mathrm{SO}$ varies significantly from 0 to $25 \%$ in older adults between studies depending on study populations and the definitions of SO. However, considering all studies together found above, the approximate average prevalence of SO in older adults is about $5-10 \%$, and it is similar between men and women. In general, the SO prevalence is lower $(3-8 \%)$ using height adjusted ALM index $[4,5,60,61]$ compared with weight or BMI adjusted ALM index $(6-10 \%)$ [20,21,56,57] in defining sarcopenia, as indicated earlier in the prevalence of sarcopenia $[16,33,50]$. Also, the $S O$ prevalence is significantly higher in people aged $\geq 80$ years, compared with that in older adults aged $<80$ years. However, SO prevalence was higher (16-25\%) when a more arbitrary definition of SO was used such as the lowest two quintiles of muscle mass or the highest two quintiles of $\%$ body fat $[22,23]$.
Physical activity \& sarcopenic obesity

Regular physical activity, including both aerobic and resistance exercise, is a significant modifiable factor for the prevention and treatment of obesity in the general population or sarcopenia in older adults. Although physical activity has generally been shown to prevent weight gain and reduce fat mass in people with obesity, while also improving muscle mass and strength in sarcopenic older people, there are still very limited data regarding the benefit of physical activity in individuals with SO. Table 1 shows the summary of current studies that have investigated the associations of physical activity, physical fitness or exercise training with SO in older adults.

In the Quebec Longitudinal Study involving 904 older adults (mean age: 74 years), the authors investigated the associations between $\mathrm{SO}$ and physical fitness using objective measures of body composition and physical fitness in independent older adults [19]. In both men and women, they found that both sarcopenic obese and nonsarcopenic obese groups had lower physical fitness levels (measured by timed up and go, chair stand, walking speed and leg stand), compared with nonsarcopenic normal weight individuals after adjusting for age, physical activity and the sum of medical conditions such as cardiac problems, stroke, diabetes, lung diseases, digestive diseases, arthritis and osteoporosis. However, the sarcopenic obese group had similar fitness levels compared with the nonsarcopenic obese group. Therefore, obesity rather than sarcopenia 
appears to contribute more to lower physical fitness in these well-functioning older men and women.

In the Korean study of 2221 elderly population (mean age: 70 years), investigators examined the associations of exercise and walking with SO [20]. Although all results did not reach statistical significance, they reported that men who participated in resistance exercise $\geq 3$ times/week, flexibility exercise $\geq 3$-times/week or walking $\geq 1 \mathrm{~h} /$ day had 53,30 and $49 \%$ lower odds of SO, respectively, compared with no resistance exercise, no flexibility exercise or walking $<30 \mathrm{~min} /$ day, respectively, after adjusting for age. Similar but slightly weaker associations were found in women. In addition, high serum insulin was significantly associated with increased risk of SO, whereas high vitamin $\mathrm{D}$ level was associated with lower risk of SO among metabolic and nutritional factors. In another Korean study, investigators used a nationally representative noninstitutionalized elderly sample of 2264 older adults aged $\geq 65$ years (mean age: 78 years) to examine the association between physical activity and SO. They found that men participating in moderate (e.g., $\geq 600$ MET-min/week) and high (e.g., $\geq 3000$ MET-min/week) physical activity had
51 and 75\% significantly lower odds of SO, respectively, compared with low activity that did not meet the moderate or high activity criteria [21]. These results were adjusted for age, education level, lifestyle factors (smoking and alcohol) and medical conditions (heart disease, stroke, diabetes, hypertension and hyperlipidemia). In women, only high, but not moderate, physical activity was associated with $57 \%$ lower odds of SO, compared with low activity in the same analysis model. Therefore, this study suggested that there is a gender difference in the relationship between physical activity and $\mathrm{SO}$, with stronger associations in men than in women. However, these differences may be due to different physical activity patterns between men and women, rather than biologically driven. For example, men tend to engage in more vigorous-intensity sporting activity and resistance exercise, whereas women perform more light-intensity domestic activities.

In the Spanish study of 306 octogenarians (mean age: 83 years), physical fitness predicted the risk of $\mathrm{SO}$ in both men and women [22]. Specifically, leg and arm strengths, agility, walking speed and balance in men, and agility and balance in women were more strongly associated with SO. Another larger Spanish study in

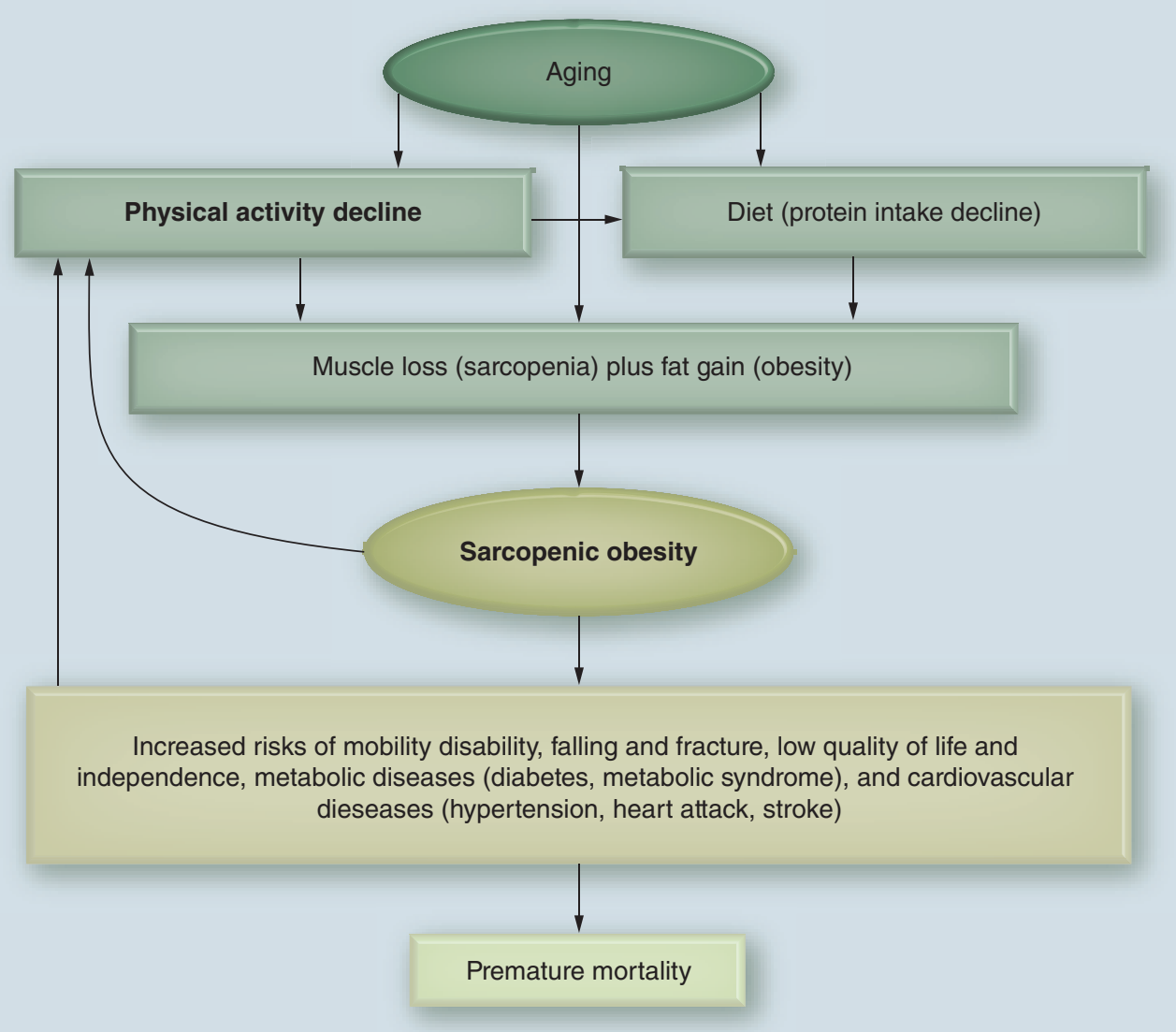

Figure 3. Relationship between physical activity and sarcopenic obesity. 
2747 noninstitutionalized elderly population (mean age: 72 years) reported similar findings indicating that lower physical fitness levels were associated with an increased risk of SO [23]. Further, balance, aerobic capacity and walking speed were identified as the most sensitive fitness tests associated with the risk of $\mathrm{SO}$ with $70-80 \%$ significantly lower odds of SO in the highest tertile compared with the lowest tertile of each fitness test score in each gender after adjusting for age. However, body strength and flexibility were less related to the risk of SO with $20-70 \%$ lower odds of SO in the highest tertile compared with the lowest tertile of each fitness test score in both men and women.

We found only one randomized controlled trial that investigated the effects of a 15 -week resistance training on physical function in 17 US older adults aged 60-90 years (mean age: 71 years) with $S O$ at baseline [24]. Physical function was assessed using the short physical performance battery test including 4-m usual gait speed, chair stand and standing balance tests. Participants were randomly assigned either traditional strength/hypertrophy (SH) training or high-speed circuit (HSC) power training. The traditional $\mathrm{SH}$ training group performed three sets of $10-12$ repetitions using $70 \%$ of their one repetition maximum on 11 exercises with 1-2 min of recovery between sets. The participants were instructed to perform the concentric and eccentric phases of each exercise in $2 \mathrm{~s}$. The HSC power training group performed the same sets and repetitions using relatively lower intensity (lower weight) with no recovery between sets. Also, the participants were instructed to perform the concentric phases of each exercise as fast as possible, and perform the eccentric phase in $2 \mathrm{~s}$. Investigators found a significant $20 \%$ improvement in physical function in the HSC power training, and a nonsignificant $7 \%$ improvement in the traditional SH training group. However, they found significant improvements in muscle strength and power using leg and chest press tests in both resistance training groups. This study clearly suggests the treatment effect of resistance exercise in SO patients, specifically that high-speed muscle power training (moving resistance at higher velocities) could be more beneficial to improve physical function. However, due to the small sample size $(\mathrm{n}=17)$ and a short intervention ( 15 weeks), additional research on this topic is warranted.

All six studies suggested potential health benefits of physical activity, fitness and resistance exercise training on SO in older adults in different populations, although the strength of the associations were different between studies. Therefore, the results between studies must be interpreted with caution, and there are several factors affecting the differences among studies such as different methods to measure physical activity, fitness or body composition; different cut-point values to categorize individuals with sarcopenia and/or obesity; age and health conditions of the study populations and different study design. Five out of six studies used a cross-sectional study design, and there is only one small exercise intervention study with a short training period of 15 weeks. Therefore, causality between physical activity and $\mathrm{SO}$ cannot be established in most studies, and the effect of changes in physical activity over time on SO remains to be determined. Thus, longitudinal studies are needed to investigate long-term effects of physical activity and fitness on SO in older individuals. Also, more studies are needed to establish the most effective type or combination of exercise and its optimal amount and intensity for the prevention and management of SO. Furthermore, to minimize unfavorable adverse events, exercise prescription should consider the higher risk of injury or complications in the elderly due to clinical conditions such as osteoporosis or cardiometabolic diseases.

In addition to physical activity, diet including adequate protein intake and amino acid supplementation is another key factor in effectively enhancing body composition, as recommended for the management of sarcopenia and $S O[17,18,62,63]$. In a recent study, exercise combined with amino acid supplementation was most effective in improving muscle mass, strength and walking speed in women with sarcopenia [64]. Another strategy besides exercise and diet could include a pharmaceutical approach such as hormone therapy (growth hormone), since low levels of growth hormone are a risk factor for sarcopenia [65]. SO is a continuous process for years and decades, although it progresses and accelerates faster after the age of 60 . Therefore, multiple lifelong approaches combining physical activity, diet and potentially pharmaceutical interventions should be considered for the prevention and treatment of SO.

\section{Mechanisms linking physical activity to sarcopenic obesity}

SO is a multifaceted medical condition with a complicated etiology and results in varying health consequences. The central cause of $\mathrm{SO}$ in older adults is the age-related decline in muscle mass and accumulation of fat mass, directly or indirectly through changes in physical activity and diet, as illustrated in Figure 3. SO, however, can also occur in young adults. For example, due to repetitive extreme diet for weight loss (yo-yo diet) following excessive caloric restriction and unbalanced diet, which may cause muscle loss, weight regain and obesity [66]. However, fat distribution may be different in SO in older adults with more intramuscular (fat infiltration into muscle) [28,67] and visceral fat increase and subcutaneous fat decrease with aging [68]. 
Possible reasons for the decline in aerobic and musclestrengthening physical activity by aging include a decreased occupational activity after retirement, osteoarthritis and muscular skeletal injuries, fear of falling and fracture risk due to osteoporosis and decreased interest and physical ability in sports and exercise. In addition, decreased appetite and gastrointestinal function by aging may also reduce protein intake and digestion, which attributes to muscle loss due to decreased muscle protein synthesis [69], leading to sarcopenia and SO.

$\mathrm{SO}$ is associated with or leads to several adverse outcomes with increased risks of mobility disability [4-6], falling and fracture [70], low quality of life and independence [49], metabolic diseases [7,8] and cardiovascular diseases [9-11], which increases risk of premature mortality [12-14]. Potential mechanisms for the development of $\mathrm{SO}$ and its health problems include increased insulin resistance [20,56], increased chronic inflammation [58,71], decreased hormones (testosterone, growth hormone, DHEA, IFG-1) [72], decreased neuromuscular function [73-75] and decreases in energy expenditure and fat oxidation [76].

$\mathrm{SO}$ and chronic diseases may also cause physical inactivity due to reduced exercise capacity (decreased cardiorespiratory fitness and muscular strength), physical limitations [77] and increased fatigue after exercise. Therefore, breaking this vicious cycle by increasing physical activity and sufficient protein intake should be emphasized to prevent $\mathrm{SO}$ and its related health consequences.

\section{Discussion (current limitations \& future direction)}

Current sarcopenia and SO definitions have been primarily developed based on data available from crosssectional analyses, and there is still no universal definition with consensus. This lack of unified definition for $\mathrm{SO}$ has contributed inconsistent findings on the prevalence of $\mathrm{SO}$ and the associations between $\mathrm{SO}$ and health outcomes. Therefore, it is important to continue to discuss and develop consensus criteria for a clinical definition of SO by comparing different definitions in various populations including those with increased risk of functional disability, bone fracture, joint replacement surgery and other musculoskeletal injuries. It is also important to measure changes in $\mathrm{SO}$ variables over time in the same individuals. Therefore, longitudinal studies and randomized controlled trials should be performed to investigate the causation and prognosis of SO. These studies can contribute to finding the effects of changes in ALM, muscle strength, gait speed and fat mass on SO. For clinical trials, including the primary SO parameters (muscle mass, muscle strength and physical function, fat mass and waist circumference) as well as relevant clinical outcomes such as cardiometabolic markers, chronic inflammatory markers, quality of life, falling and fracture, activities of daily living and independence (hospitalization) should be considered to provide evidence based SO prevention and treatment strategies.

Based on the significant differences in body composition and muscle strength between men and women, sarcopenia definitions for muscle mass and strength were presented separately by gender. However, regarding gait speed, the same cut-point value is used to define poor physical function, although the value is different between men and women as presented in USA [33] and the Asian groups [32] on sarcopenia. Therefore, further study may be warranted addressing the question whether different cut-points of gait speed for men and women should be utilized in accordance to other measures of physical function. Regarding muscle strength, the current recommendation is to use unadjusted handgrip strength because it is simple to use clinically, although handgrip strength is highly correlated with body weight. The FNIHSP study also reported that BMI adjusted ALM index had consistent associations with mobility limitations between different study samples in women [78]. However, continuous clarification and further investigation is needed if body weight or BMI adjusted handgrip strength or muscle strength should be used based on its relations to various health outcomes, as used in other studies on muscle strength and health outcomes [43-45]. In addition, while gait speed and handgrip strength are easy to measure, more practical and comprehensive assessment of functional ability and total body strength such as the sitting-rising test [79] should be developed. Those new tests should be related to daily activities and health outcomes such as quality of life and mortality in older adults, and could be easily measured by individuals at home without using instruments.

In defining and diagnosing sarcopenia, the current cut-point values for muscle mass were mostly developed based on the lowest $20 \%$ or two SDs below the mean value of young healthy reference group from a specific study population. This method is originally designed to parallel the definition of osteoporosis (reduced bone mass), which has been associated with risk of fracture. However, low muscle mass alone is not consistently associated with relevant adverse health outcomes. Therefore, more studies are urgently needed to have reliable and validated reference values for low muscle mass in general populations around the world, as suggested by the FNIHSP [33]. In addition, the cutpoint values should preferably be developed based on prospective outcome studies, instead of those simple statistical methods (the lowest $20 \%$ or two SDs below the mean value of the reference group). 
BMI is easy to measure and commonly used to define obesity worldwide as a representative measure of whole body obesity in population levels. However, because BMI does not consider body composition such as muscle and fat mass, its accuracy in research is continuously debated, especially in older adults and specifically in the assessment of SO. Compared with young adults, older adults could have more fat mass given the same BMI levels due to muscle loss by aging. Therefore, it is possible that older adults even in the normal weight category by BMI could have more fat mass, which increases the risk of cardiometabolic diseases such as diabetes or heart attack. Future studies on SO should measure body composition instead of BMI to define SO in older adults. Additionally, because obesity is simply defined by BMI without considering metabolic health and fitness, there are controversial issues regarding different obesity phenotypes such as metabolically healthy obesity [80], and obesity with high cardiorespiratory fitness levels [81,82] that may not be associated with increased risks of cardiometabolic diseases and mortality. There is also an obesity paradox indicating that obesity has some mortality benefits in individuals with heart diseases or Type II diabetes [83,84]. Also, a recent review reported mortality benefits in overweight and obesity in older adults $[85,86]$, which is another obesity paradox in older adults. The answers to these controversial issues on different obesity phenotypes and paradox may be related to muscle mass and strength, especially in older adults. Therefore, future studies on obesity and SO should consider more comprehensive approaches including body composition and muscle mass in the analyses.

Another type of muscle disorder that should be studied is dynapenia, which is more closely related to muscle quality rather than muscle quantity (muscle mass). Possible causes of dynapenia include impairments in neural activation, decreased muscle contractile quality and reduced motor unit recruitment [74], which leads to significant loss of muscle strength. Loss of muscle mass due to sarcopenia also affects dynapenia. Several studies indicated that older adults with dynapenia have increased risk of functional disability [87], falling [70] and metabolic diseases [88], even after adjusting for sarcopenia [89]. In our earlier study, we found that individuals with lower muscle strength (the lowest 20th percentile) compared with the moderate or high muscle strength (upper 80th percentile) had over twofold higher risk of developing metabolic syndrome in both young ( $<50$ years) and old ( $\geq 50$ years) men after adjusting for age, smoking and alcohol intake [90]. This association remained significant, although reduced, even after further adjustment for BMI and cardiorespiratory fitness in both young and old men. We also found that individuals with higher muscle strength had lower risk of developing obesity [44] and reduced allcause, CVD and cancer mortality regardless of their body fatness [45,91]. Further, even hypertensive men with higher muscle strength (upper third) had 34\% lower risk of all-cause mortality compared with lower muscle strength (lower third), after adjusting for body fatness and cardiorespiratory fitness [43]. These findings clearly support the importance of muscle strength as an independent risk factor for chronic diseases and mortality not only in older but also in general populations. However, there are still relatively limited data on muscle strength and resistance exercise on health outcomes compared with cardiorespiratory fitness and aerobic exercise on health. Therefore, more studies are required on this important topic of resistance exercise and muscle strength in relation to dynapenia, sarcopenia and SO.

There are also several important questions that should be considered in relation to physical activity and SO. What type and amount of physical activity and exercise are most effective for SO prevention and treatment in older adults with and without functional limitations? What are the practical strategies to promote and increase habitual physical activity in older adults? How can physical activity be combined with other lifestyle factors including sufficient protein intake, healthy diet and chronic disease management for prevention and treatment of SO?

\section{Conclusion \& future perspective}

Based on the current review, the average prevalence of $\mathrm{SO}$ in older adults ranges from 5 to $10 \%$ depending on its definitions and study populations, and the prevalence is significantly higher in people aged $\geq 80$ years. The number of older adults aged $\geq 60$ years is expected to more than double, from 841 million in 2013 to over 2 billion in 2050 globally [3]. Accordingly, the percentage of older adults is expected to increase from $11.7 \%$ in 2013 to $21.1 \%$ by 2050 . This older population is also aging themselves, and the proportion of the oldest old people aged $\geq 80$ years is projected to increase dramatically. Further, older adults are projected to exceed the number of children for the first time in 2047 worldwide [3]. Based on the above estimation of the prevalence of $\mathrm{SO}$ and older adult population, SO may affect 40-80 million people today and will affect 100-200 million in the next 35 years globally.

Sarcopenia and SO studies have emerged only in the past 2-3 decades, and research in this area is still in its infancy. However, the population with SO around the world has major health, social and economic consequences, often with limited coverage of social security 
system in many developing countries [3]. Therefore, most countries are expected to experience increasing challenges to deal with the significant impact of SO regarding mobility disability, hospitalization, increased chronic diseases, mortality and healthcare cost.

Although we found many important questions that remain unanswered, it is important to note that $\mathrm{SO}$ can be prevented, delayed and treated by maintaining or adopting a healthy lifestyle, including increasing aerobic and muscle-strengthening activities and sufficient protein intake and healthy diet. Specifically, we found potential health benefits of physical activity, fitness and resistance exercise training on $\mathrm{SO}$ in older adults in different populations in this review (Table 1). However, most findings are based on cross-sectional studies. Therefore, longitudinal studies are clearly needed to investigate long-term effects of physical activity and fitness on SO in older individuals. We hope that the current review guides researchers and health professionals around the world to further refine definition, diagnosis and classification of sarcopenia and SO for both research studies and clinical practice. It is also important to develop simple and easy measurement techniques and tools that are reliable and valid, thus it can be included in the routine geriatric assessment to provide targeted and appropriate intervention. Simultaneously, we should also continue to identify effective public health strategies and programs to combat $S O$ for health promotion and quality of life improvement for millions of older people around the world.

\section{Financial \& competing interests disclosure}

SN Blair has served on the scientific advisory boards of Technogym, Clarity, Cancer Foundation for Life, and Santech. He has received research funding from BodyMedia, Technogym, The Coca-Cola Company, the US Department of Defense, and the NIH. He receives book royalties from Human Kinetics. The authors have no other relevant affiliations or financial involvement with any organization or entity with a financial interest in or financial conflict with the subject matter or materials discussed in the manuscript apart from those disclosed.

No writing assistance was utilized in the production of this manuscript.

\begin{abstract}
Author contributions
The first author, DC Lee, designed, developed and drafted the manuscript. All other authors critically reviewed the manuscript including the text, figures and tables, and provided significant comments.
\end{abstract}

\section{Open access}

This work is licensed under the Creative Commons Attribution 4.0 License. To view a copy of this license, visit http://creativecommons.org/licenses/by/4.0/

\section{Executive summary}

- Sarcopenic obesity (SO) is the combination of low muscle mass plus high fat mass based on the body composition phenotypes. However, modern definition of sarcopenia includes low muscle mass, low muscle strength (weak handgrip strength) and poor physical function (slow walking).

- Based on the sarcopenia definitions suggested by different working groups, the cut-points for low muscle mass for men and women using appendicular lean mass divided by height ${ }^{2}$ are $\leq 7.0 \mathrm{~kg} / \mathrm{m}^{2}$ and $\leq 5.4 \mathrm{~kg} / \mathrm{m}^{2}$ in Asians, and $\leq 7.23 \mathrm{~kg} / \mathrm{m}^{2}$ and $\leq 5.67 \mathrm{~kg} / \mathrm{m}^{2}$ in Caucasians, respectively. The recommended cut-points for weak handgrip strength for men and women are $<26 \mathrm{~kg}$ and $<18 \mathrm{~kg}$ in Asians, and $<30 \mathrm{~kg}$ and $<20 \mathrm{~kg}$ in Caucasians, respectively. The most common cut-points for slow walking is $\leq 0.8 \mathrm{~m} / \mathrm{s}$ in both men and women regardless of race and ethnicity. However, there is a wide range of variations in the definition, assessment and diagnosis of SO for both studies.

- Although the prevalence of SO varies significantly from 0 to $25 \%$ in older adults between studies depending on study populations and the definitions of SO, the average prevalence of SO is about $5-10 \%$, which is similar between men and women. The prevalence of SO is significantly higher in people aged $\geq 80$ years.

- Older adults with SO have higher risks of mobility disability, metabolic diseases, hypertension, cardiovascular diseases and mortality, which is also related to significantly higher healthcare cost. These clinical problems related to SO is much greater than that in sarcopenia or obesity alone.

- Despite the limited data, current studies suggest that there are potential health benefits of physical activity, fitness and exercise training on the prevention and treatment of SO in older adults. Sufficient diet such as adequate protein intake and amino acid supplementation is another key factor for the management of SO.

- Potential mechanisms on the development of SO and its health problems include increased insulin resistance, increased chronic inflammation, decreased hormones, decreased neuromuscular function and decreased energy expenditure and fat oxidation due to aging.

- Given the rapidly increasing health impact of SO in aging populations in most developed countries, the current review highlights the importance of the urgent investigation and development of universal definition, assessment and diagnosis of SO for both research studies and clinical practice. In addition, we should continue to identify effective public health strategies and programs to prevent, delay and treat SO for millions of older people around the world. 


\section{References}

1 Rosenberg IH. Sarcopenia. origins and clinical relevance. J. Nutr. 127(Suppl. 5), S990-S999 (1997).

2 Ogden CL, Carroll MD, Kit BK, Flegal KM. Prevalence of childhood and adult obesity in the United States, 2011-2012. JAMA 311(8), 806-814 (2014).

3 United Nations, Department of Economic and Social Affairs, Population Division. World population ageing (2013).

www.un.org/

4 Baumgartner RN, Wayne SJ, Waters DL, Janssen I, Gallagher D, Morley JE. Sarcopenic obesity predicts instrumental activities of daily living disability in the elderly. Obes. Res. 12(12), 1995-2004 (2004).

5 Rolland Y, Lauwers-Cances V, Cristini C et al. Difficulties with physical function associated with obesity, sarcopenia, and sarcopenic-obesity in community-dwelling elderly women. the EPIDOS (EPIDemiologie de l'OSteoporose) study. Am. J. Clin. Nutr. 89(6), 1895-1900 (2009).

6 Stenholm S, Alley D, Bandinelli S et al. The effect of obesity combined with low muscle strength on decline in mobility in older persons. Results from the InCHIANTI study. Int. J. Obes. (Lond.) 33(6), 635-644 (2009).

7 Kim TN, Park MS, Yang SJ et al. Prevalence and determinant factors of sarcopenia in patients with Type 2 diabetes. The Korean Sarcopenic Obesity Study (KSOS). Diabetes Care 33(7), 1497-1499 (2010).

8 Lim S, Kim JH, Yoon JW et al. Sarcopenic obesity. Prevalence and association with metabolic syndrome in the Korean Longitudinal Study on Health and Aging (KLoSHA). Diabetes Care 33(7), 1652-1654 (2010).

9 Park SH, Park JH, Song PS et al. Sarcopenic obesity as an independent risk factor of hypertension. J. Am. Soc. Hypertens. 7(6), 420-425 (2013).

10 Kim TN, Choi KM. The implications of sarcopenia and sarcopenic obesity on cardiometabolic disease. J. Cell Biochem. 116(7), 1171-1178 (2015).

11 Stephen WC, Janssen I. Sarcopenic-obesity and cardiovascular disease risk in the elderly. J. Nutr. Health Aging 13(5), 460-466 (2009).

12 Atkins JL, Whincup PH, Morris RW, Lennon LT, Papacosta O, Wannamethee SG. Sarcopenic obesity and risk of cardiovascular disease and mortality. A population-based cohort study of older men. J. Am. Geriatr. Soc. 62(2), 253-260 (2014).

13 Chuang SY, Hsu YY, Chen RC, Liu WL, Pan WH. Abdominal obesity and low skeletal muscle mass jointly predict total mortality and cardiovascular mortality in an elderly Asian population. J. Gerontol. A Biol. Sci. Med. Sci. (2015) (Epub ahgead of print).

14 Tian S, Xu Y. Association of sarcopenic obesity with the risk of all-cause mortality. A meta-analysis of prospective cohort studies. Geriatr. Gerontol. Int. 16(2), 155-166 ( 2015).

15 Janssen I, Shepard DS, Katzmarzyk PT, Roubenoff R. The healthcare costs of sarcopenia in the United States. J. Am. Geriatr. Soc. 52(1), 80-85 (2004).
16 Cauley JA. An overview of sarcopenic obesity. J. Clin. Densitom. 18(4), 499-505 (2015).

17 Goisser S, Kemmler W, Porzel S et al. Sarcopenic obesity and complex interventions with nutrition and exercise in community-dwelling older persons - a narrative review. Clin. Interv. Aging 10, 1267-1282 (2015).

18 Poggiogalle E, Migliaccio S, Lenzi A, Donini LM. Treatment of body composition changes in obese and overweight older adults. Insight into the phenotype of sarcopenic obesity. Endocrine 47(3), 699-716 (2014).

19 Bouchard DR, Dionne IJ, Brochu M. Sarcopenic/obesity and physical capacity in older men and women. Data from the Nutrition as a Determinant of Successful Aging (NuAge) the Quebec longitudinal study. Obesity (Silver Spring) 17(11), 2082-2088 (2009).

20 Hwang B, Lim JY, Lee J, Choi NK, Ahn YO, Park BJ. Prevalence rate and associated factors of sarcopenic obesity in Korean elderly population. J. Korean Med. Sci. 27(7), 748-755 (2012).

21 Ryu M, Jo J, Lee Y, Chung YS, Kim KM, Baek WC. Association of physical activity with sarcopenia and sarcopenic obesity in community-dwelling older adults. The Fourth Korea National Health and Nutrition Examination Survey. Age Ageing 42 (6), 734-740 (2013).

22 Munoz-Arribas A, Mata E, Pedrero-Chamizo R et al. [Sarcopenic obesity and physical fitness in octogenarians. The multi-center EXERNET project]. Nutr. Hosp. 28(6), 1877-1883 (2013)

23 Pedrero-Chamizo R, Gomez-Cabello A, Melendez A et al. Higher levels of physical fitness are associated with a reduced risk of suffering sarcopenic obesity and better perceived health among the elderly. The EXERNET multi-center study. J. Nutr. Health Aging 19(2), 211-217 (2015).

24 Balachandran A, Krawczyk SN, Potiaumpai M, Signorile JF. High-speed circuit training vs hypertrophy training to improve physical function in sarcopenic obese adults. A randomized controlled trial. Exp. Gerontol. 60, 64-71 (2014).

25 Leong DP, Teo KK, Rangarajan S et al. Prognostic value of grip strength. Findings from the Prospective Urban Rural Epidemiology (PURE) study. Lancet 386(9990), 266-273 (2015).

26 Abellan Van KG, Rolland Y, Andrieu S et al. Gait speed at usual pace as a predictor of adverse outcomes in communitydwelling older people an International Academy on Nutrition and Aging (IANA) Task Force. J. Nutr. Health Aging 13(10), 881-889 (2009)

27 Studenski S, Perera S, Patel K et al. Gait speed and survival in older adults. JAMA 305(1), 50-58 (2011).

28 Visser M, Goodpaster BH, Kritchevsky SB et al. Muscle mass, muscle strength, and muscle fat infiltration as predictors of incident mobility limitations in wellfunctioning older persons. J. Gerontol. A Biol. Sci. Med. Sci. 60(3), 324-333 (2005).

29 Newman AB, Kupelian V, Visser M et al. Strength, but not muscle mass, is associated with mortality in the health, aging and body composition study cohort. J. Gerontol. A Biol. Sci. Med. Sci. 61(1), 72-77 (2006). 
30 Cruz-Jentoft AJ, Baeyens JP, Bauer JM et al. Sarcopenia. European consensus on definition and diagnosis: report of the European Working Group on Sarcopenia in older people. Age Ageing 39 (4), 412-423 (2010).

31 Fielding RA, Vellas B, Evans WJ et al. Sarcopenia. An undiagnosed condition in older adults. Current consensus definition: prevalence, etiology, and consequences. International Working Group on Sarcopenia. J. Am. Med. Dir. Assoc. 12(4), 249-256 (2011).

32 Chen LK, Liu LK, Woo J et al. Sarcopenia in Asia. Consensus report of the Asian Working Group for Sarcopenia. J. Am. Med. Dir. Assoc. 15(2), 95-101 (2014).

33 Studenski SA, Peters KW, Alley DE et al. The FNIH sarcopenia project. Rationale, study description, conference recommendations, and final estimates. J. Gerontol. A Biol. Sci. Med. Sci. 69(5), 547-558 (2014).

34 Chien MY, Huang TY, Wu YT. Prevalence of sarcopenia estimated using a bioelectrical impedance analysis prediction equation in community-dwelling elderly people in Taiwan. J. Am. Geriatr. Soc. 56(9), 1710-1715 (2008).

35 Janssen I, Baumgartner RN, Ross R, Rosenberg IH, Roubenoff R. Skeletal muscle cutpoints associated with elevated physical disability risk in older men and women. Am. J. Epidemiol. 159(4), 413-421 (2004).

36 Baumgartner RN, Koehler KM, Gallagher D et al. Epidemiology of sarcopenia among the elderly in New Mexico. Am. J. Epidemiol. 147(8), 755-763 (1998).

37 Ido A, Nakayama Y, Ishii K et al. Ultrasound-derived abdominal muscle thickness better detects metabolic syndrome risk in obese patients than skeletal muscle index measured by dual-energy x-ray absorptiometry. PLoS ONE 10(12), e0143858 (2015).

38 Akin S, Mucuk S, Ozturk A et al. Muscle functiondependent sarcopenia and cut-off values of possible predictors in community-dwelling Turkish elderly. Calf circumference, midarm muscle circumference and walking speed. Eur. J. Clin. Nutr. 69(10), 1087-1090 (2015).

39 Kawakami R, Murakami H, Sanada K et al. Calf circumference as a surrogate marker of muscle mass for diagnosing sarcopenia in Japanese men and women. Geriatr. Gerontol. Int. 15(8), 969-976 (2015).

40 Janssen I, Heymsfield SB, Ross R. Low relative skeletal muscle mass (sarcopenia) in older persons is associated with functional impairment and physical disability. J. Am. Geriatr. Soc. 50 (5), 889-896 (2002).

41 Cuoco A, Callahan DM, Sayers S, Frontera WR, Bean J, Fielding RA. Impact of muscle power and force on gait speed in disabled older men and women. J. Gerontol. A Biol. Sci. Med. Sci. 59(11), 1200-1206 (2004).

42 Herman S, Kiely DK, Leveille S, O’Neill E, Cyberey S, Bean JF. Upper and lower limb muscle power relationships in mobility-limited older adults. J. Gerontol. A Biol. Sci. Med. Sci. 60(4), 476-480 (2005).

43 Artero EG, Lee DC, Ruiz JR et al. A prospective study of muscular strength and all-cause mortality in men with hypertension. J. Am. Coll. Cardiol. 57(18), 1831-1837 (2011).
44 Jackson AW, Lee DC, Sui X et al. Muscular strength is inversely related to prevalence and incidence of obesity in adult men. Obesity (Silver Spring) 18(10), 1988-1995 (2010).

45 Ruiz JR, Sui X, Lobelo F et al. Association between muscular strength and mortality in men. Prospective cohort study. BMJ 337( a 439), 1-9 (2008).

46 Afilalo J, Eisenberg MJ, Morin JF et al. Gait speed as an incremental predictor of mortality and major morbidity in elderly patients undergoing cardiac surgery. J. Am. Coll. Cardiol. 56(20), 1668-1676 (2010).

47 Guralnik JM, Ferrucci L, Simonsick EM, Salive ME, Wallace RB. Lower-extremity function in persons over the age of 70 years as a predictor of subsequent disability. N. Engl. J. Med. 332(9), 556-561 (1995).

48 Toots A, Rosendahl E, Lundin-Olsson L, Nordstrom P, Gustafson Y, Littbrand H. Usual gait speed independently predicts mortality in very old people. A population-based study. J. Am. Med. Dir. Assoc. 14(7), 529.e1-529.e6 (2013).

49 Goodpaster BH, Park SW, Harris TB et al. The loss of skeletal muscle strength, mass, and quality in older adults. The health, aging and body composition study. J. Gerontol. A Biol. Sci. Med. Sci. 61(10), 1059-1064 (2006).

50 Dam TT, Peters KW, Fragala M et al. An evidence-based comparison of operational criteria for the presence of sarcopenia. J. Gerontol. A Biol. Sci. Med. Sci. 69(5), 584-590 (2014).

51 Newman AB, Kupelian V, Visser M et al. Sarcopenia. Alternative definitions and associations with lower extremity function. J. Am. Geriatr. Soc. 51(11), 1602-1609 (2003).

$52 \mathrm{Kim}$ M, Kim H. Accuracy of segmental multi-frequency bioelectrical impedance analysis for assessing whole-body and appendicular fat mass and lean soft tissue mass in frail women aged 75 years and older. Eur. J. Clin. Nutr. 67(4), 395-400 (2013).

53 Yeh CW, Chen YJ, Lai LY et al. Bioelectrical impedance analysis in a mathematical model for estimating fat-free mass in multiple segments in elderly Taiwanese males. Int. J. Gerontol. 6(4), 273-277 (2012).

54 WHO Expert Consultation. Appropriate body-mass index for Asian populations and its implications for policy and intervention strategies. Lancet 363(9403), 157-163 (2004).

55 World Health Organization, International Association for the Study of Obesity, International Obesity Task Force. The Asia-Pacific perspective. redefining obesity and its treatment. Sydney. Health Communication, Australia (2000). www.wpro.who.int/

56 Levine ME, Crimmins EM. The impact of insulin resistance and inflammation on the association between sarcopenic obesity and physical functioning. Obesity (Silver Spring) 20(10), 2101-2106 (2012).

57 Kim YS, Lee Y, Chung YS et al. Prevalence of sarcopenia and sarcopenic obesity in the Korean population based on the Fourth Korean National Health and Nutritional Examination Surveys. J. Gerontol. A Biol. Sci. Med. Sci. 67(10), 1107-1113 (2012). 
58 Schrager MA, Metter EJ, Simonsick E et al. Sarcopenic obesity and inflammation in the InCHIANTI study. J. Appl. Physiol. (1985) 102(3), 9-925 (2007).

59 Stenholm S, Harris TB, Rantanen T, Visser M, Kritchevsky SB, Ferrucci L. Sarcopenic obesity. Definition, cause and consequences. Curr. Opin. Clin. Nutr. Metab. Care 11(6), 693-700 (2008).

60 Meng P, Hu YX, Fan L et al. Sarcopenia and sarcopenic obesity among men aged 80 years and older in Beijing. Prevalence and its association with functional performance. Geriatr. Gerontol. Int. 14(Suppl. 1), 29-35 (2014).

61 Dufour AB, Hannan MT, Murabito JM, Kiel DP, McLean RR. Sarcopenia definitions considering body size and fat mass are associated with mobility limitations. The Framingham Study. J. Gerontol. A Biol. Sci. Med. Sci. 68(2), 168-174 (2013).

62 Li Z, Heber D. Sarcopenic obesity in the elderly and strategies for weight management. Nutr. Rev. 70(1), 57-64 (2012).

63 Morley JE, Argiles JM, Evans WJ et al. Nutritional recommendations for the management of sarcopenia. J. Am. Med. Dir. Assoc. 11(6), 3-396 (2010).

64 Kim HK, Suzuki T, Saito K et al. Effects of exercise and amino acid supplementation on body composition and physical function in community-dwelling elderly Japanese sarcopenic women. A randomized controlled trial. J. Am. Geriatr. Soc. 60(1), 16-23 (2012).

65 Bouchonville MF, Villareal DT. Sarcopenic obesity. How do we treat it? Curr. Opin. Endocrinol. Diabetes Obes. 20(5), 412-419 (2013).

66 Dulloo AG, Montani JP. Pathways from dieting to weight regain, to obesity and to the metabolic syndrome. An overview. Obes. Rev. 16(Suppl. 1), 1-6 (2015).

67 Song MY, Ruts E, Kim J, Janumala I, Heymsfield S, Gallagher D. Sarcopenia and increased adipose tissue infiltration of muscle in elderly African American women. Am. J. Clin. Nutr. 79(5), 874-880 (2004).

68 Hughes VA, Roubenoff R, Wood M, Frontera WR, Evans WJ, Fiatarone Singh MA. Anthropometric assessment of 10 -y changes in body composition in the elderly. Am. J. Clin. Nutr. 80(2), 475-482 (2004).

69 Short KR, Nair KS. The effect of age on protein metabolism. Curr. Opin. Clin. Nutr. Metab. Care 3(1), 39-44 (2000).

70 Scott D, Daly RM, Sanders KM, Ebeling PR. Fall and Fracture risk in sarcopenia and dynapenia with and without obesity. The role of lifestyle interventions. Curr. Osteoporos. Rep. 13(4), 235-244 (2015).

71 Kob R, Bollheimer LC, Bertsch T et al. Sarcopenic obesity. Molecular clues to a better understanding of its pathogenesis? Biogerontology 16(1), 15-29 (2015).

72 Morley JE. Hormones and the aging process. J. Am. Geriatr. Soc. 51(Suppl. 7), S333-S337 (2003).

73 Aagaard P, Suetta C, Caserotti P, Magnusson SP, Kjaer M. Role of the nervous system in sarcopenia and muscle atrophy with aging. Strength training as a countermeasure. Scand. J. Med. Sci. Sports 20(1), 49-64 (2010).

74 Lexell J, Taylor CC, Sjostrom M. What is the cause of the ageing atrophy? Total number, size and proportion of different fiber types studied in whole vastus lateralis muscle from 15 to 83 year-old men. J. Neurol. Sci. 84(2), 275-294 (1988).

75 Piasecki M, Ireland A, Jones DA, McPhee JS. Agedependent motor unit remodelling in human limb muscles. Biogerontology17(3), 485-496 (2015).

76 Roubenoff R. Sarcopenic obesity. Does muscle loss cause fat gain? Lessons from rheumatoid arthritis and osteoarthritis. Ann. NY Acad. Sci. 904, 553-557 (2000).

77 Guralnik JM, Ferrucci L, Pieper CF et al. Lower extremity function and subsequent disability. Consistency across studies, predictive models, and value of gait speed alone compared with the short physical performance battery. J. Gerontol. A Biol. Sci. Med. Sci. 55(4), M221-M231 (2000).

78 McLean RR, Shardell MD, Alley DE et al. Criteria for clinically relevant weakness and low lean mass and their longitudinal association with incident mobility impairment and mortality. The foundation for the National Institutes of Health (FNIH) sarcopenia project. J. Gerontol. A Biol. Sci. Med. Sci. 69(5), 576-583 (2014).

79 Brito LB, Ricardo DR, Araujo DS, Ramos PS, Myers $\mathrm{J}$, Araujo CG. Ability to sit and rise from the floor as a predictor of all-cause mortality. Eur. J. Prev. Cardiol. 21(7), 892-898 (2014)

80 Ortega FB, Lee DC, Katzmarzyk PT et al. The intriguing metabolically healthy but obese phenotype. Cardiovascular prognosis and role of fitness. Eur. Heart J. 34(5), 389-397 (2013).

81 Lee DC, Sui X, Artero EG et al. Long-term effects of changes in cardiorespiratory fitness and body mass index on all-cause and cardiovascular disease mortality in men. The Aerobics Center Longitudinal Study. Circulation 124(23), 2483-2490 (2011).

82 Lee DC, Sui X, Church TS, Lavie CJ, Jackson AS, Blair $\mathrm{SN}$. Changes in fitness and fatness on the development of cardiovascular disease risk factors hypertension, metabolic syndrome, and hypercholesterolemia. J. Am. Coll. Cardiol. 59(7), 665-672 (2012).

83 Carnethon MR, De Chavez PJ, Biggs ML et al. Association of weight status with mortality in adults with incident diabetes. JAMA 308(6), 581-590 (2012).

84 McAuley PA, Artero EG, Sui X et al. The obesity paradox, cardiorespiratory fitness, and coronary heart disease. Mayo Clin. Proc. 87(5), 443-451 (2012).

85 Flegal KM, Kit BK, Orpana H, Graubard BI. Association of all-cause mortality with overweight and obesity using standard body mass index categories. A systematic review and meta-analysis. JAMA 309 (1), 71-82 (2013).

86 Veronese N, Cereda E, Solmi M et al. Inverse relationship between body mass index and mortality in older nursing home residents. A meta-analysis of 19,538 elderly subjects. Obes. Rev. 16(11), 1001-1015 (2015).

87 Bouchard DR, Janssen I. Dynapenic-obesity and physical function in older adults. J. Gerontol. A Biol. Sci. Med. Sci. 65(1), 71-77 (2010).

88 Senechal M, Dionne IJ, Brochu M. Dynapenic abdominal obesity and metabolic risk factors in adults 50 years of age and older. J. Aging Health 24(5), 812-826 (2012). 
89

Clark BC, Manini TM. Functional consequences of sarcopenia and dynapenia in the elderly. Curr. Opin. Clin. Nutr. Metab. Care 13(3), 271-276 (2010).

90 Senechal M, McGavock JM, Church TS et al. Cut points of muscle strength associated with metabolic syndrome in men. Med. Sci. Sports Exerc. 46(8), 1475-1481 (2014).
91 Ruiz JR, Sui X, Lobelo F et al. Muscular strength and adiposity as predictors of adulthood cancer mortality in men. Cancer Epidemiol. Biomarkers Prev. 18(5), 1468-1476 (2009). 